\title{
Tumor fibroso solitario de la pleura. Análisis de 41 casos
}

\author{
RAIMUNDO SANTOLAYA C.*, MANUEL MENESES C.**, JAVIER LÓPEZ S.****, \\ RAFAEL PRATS M.*, MAURICIO FICA D.*, CRISTIAN GONZÁLEZ C.*, ALFONSO OROPESA C.*, \\ LORETO TAPIA M.***, CARLOS CZISCHKE DEL P.* y PATRICIO RODRÍGUEZ D.*
}

\section{Solitary fibrous tumor of the pleura. Analysis about 41 cases}

Solitary fibrous tumor of the pleura (TFSP) is an uncommon and mostly benign neoplasm. It grows slowly and presents with scant symptoms. Recurrence after surgical excision has been described. The aim of this report is to show our experience in managing this disease. Clinical as well as radiological, surgical, histopathological and follow-up data were analyzed in a clinical series. Forty one cases of TFSP that underwent surgery at our institution between 1991 and 2004 were retrospectively analyzed; 27 were females and 14 males with a median age of 60.4 years old; $24.4 \%$ of the cases were asymptomatic. All tumors were surgically excised, in average they weighed $945 \mathrm{~g}$ and $36.5 \%$ of the lesions showed a pedicle. Most of the lesions were originated in the visceral pleura. Surgical morbidity was $12.1 \%$ and no mortality was recorded. Three tumor recurred. TFSP is usually a benign neoplasm but it can recur. Complete surgical excision is the treatment of choice of primary and recurrent tumors.

Key words: solitary fibrous tumor pleura, resection.

\section{Resumen}

Introducción: El tumor fibroso solitario de la pleura (TFSP) es una neoplasia infrecuente, considerada benigna, de crecimiento lento y poco sintomática pero que puede recidivar tras la resección quirúrgica. El objetivo de este estudio es mostrar la experiencia de nuestro centro con el manejo de estos tumores. Pacientes y Método: Análisis retrospectivo de 41 pacientes con TFSP intervenidos en nuestro Servicio entre 1991 y 2004. Se analizan datos clínicos, radiológicos, quirúrgicos, morbimortalidad, recurrencia y seguimiento. Resultados: De los 41 pacientes con TFSP resecados, 27 eran mujeres y 14 hombres. Edad media 60,4 años. 24,4\% de los pacientes fueron asintomáticos. Todos los pacientes fueron intervenidos quirúrgicamente y se realizó estudio histopatológico en todas las piezas. El peso promedio de los tumores fue de 954 g y 36,5\% eran tumores pediculados, el resto de base ancha. La mayoría (75,6\%) se originaban de la pleura visceral. No hubo mortalidad operatoria y la morbilidad alcanzó el 12,1\%. Tres tumores recidivaron. Conclusiones: El TFSP es una neoplasia rara generalmente benigna, pero que puede recidivar. El tratamiento es la resección quirúrgica completa y las recidivas deben ser resecadas nuevamente.

Palabras clave: Tumor fibroso solitario pleural, resección.

\footnotetext{
* Servicio Cirugía Torácica. Instituto Nacional del Tórax.

** Anatomía Patológica. Instituto Nacional del Tórax.

*** Unidad de Anatomía Patológica. Hospital del Salvador.

**** Becado de Cirugía, Universidad Austral de Chile.
} 


\section{Introducción}

Los tumores primarios de la pleura se dividen en dos grandes grupos: difusos y localizados. Los difusos, también llamados mesoteliomas malignos son de mucho peor pronóstico, tienen una conocida relación con la exposición al asbesto y son macroscópica e histológicamente diferentes al localizado. Los tumores fibrosos solitarios de la pleura (TFSP) son neoplasias raras, con aproximadamente 900 casos publicados en la literatura ${ }^{1,4,6,9,29}$. Fueron descritos inicialmente por Klemperer y Rabin en $1931^{1}$, y han recibido diversos nombres (mesotelioma benigno, fibroma subpleural, mesotelioma localizado, etc) hasta adquirir su nomenclatura actual. Hay evidencias ultraestructurales e inmunohistoquímicas tales como positividad a vimentina, y la no expresión de filamentos intermedios como citoqueratina y desmina ${ }^{2,3}$ que han permitido concluir que estas lesiones no son de origen

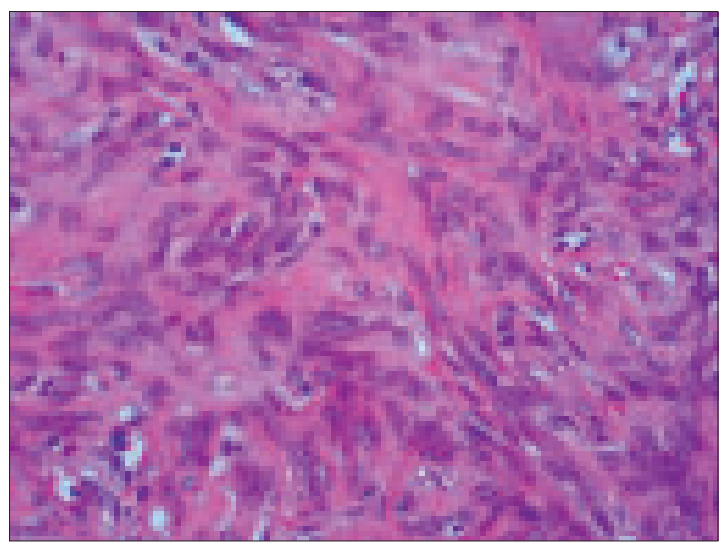

Figura 1. Tumor fusocelular de patrón verticilar típico de tumor fibroso solitario de la pleura.

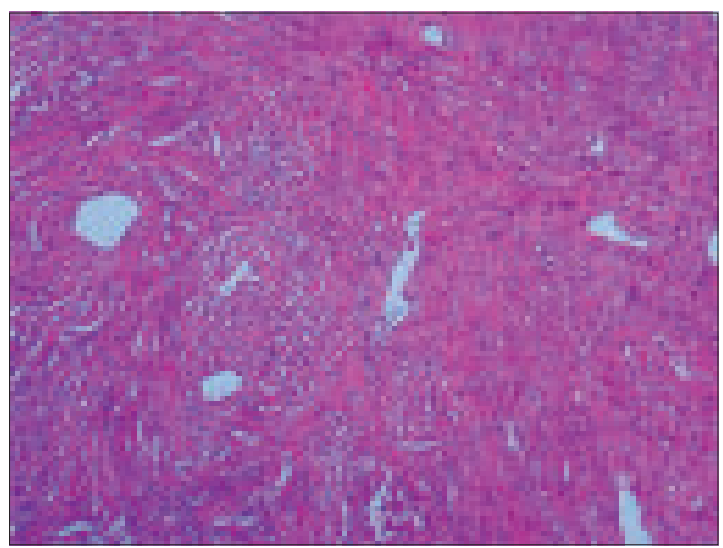

Figura 2. Tumor fibroso solitario de la pleura hipocelular. mesotelial, sino del tejido conectivo submesotelial ${ }^{4-11}$. Una característica constante es la ausencia de positividad para citoqueratinas, en contraste con la expresión constante de éstas en mesoteliomas sarcomatoides ${ }^{5}$.

Son tumores de crecimiento lento, poco sintomáticos y que pueden llegar a alcanzar grandes dimensiones. Se originan de la pleura visceral o parietal, y pueden ser pediculados o de base ancha. Se han descrito tumores de este tipo en localizaciones extratorácicas como meninges, cavidad oral, globo ocular, mama, riñón, vejiga y otros ${ }^{11-15}$. También se ha descrito hipoglicemia en relación a ellos, lo que se debería a un excesivo consumo de glucosa por la neoplasia, a sustancias similares a la insulina o a la acción de inhibidores de la neoglucogénesis segregados por el tumor ${ }^{5-7,9-11,13-19}$. Se relaciona con osteoartropatía hipertrófica en aproximadamente un $20 \%$, lo cual se debería a una anormal producción de ácido hialurónico por parte de las células tumorales que tiende a regresar con la resección del tumor ${ }^{5-7,9-11,14,20}$.

Si bien es considerada una neoplasia benigna, hay ciertas características descritas por England et $\mathrm{al}^{5}$ en una revisión de 223 casos, que sugieren como criterios de malignidad la presencia de 4 ó más mitosis por 10 campos de mayor aumento, hipercelularidad, pleomorfismo, hemorragias y necrosis tumoral. La presencia de estas características histopatológicas aumentarían la posibilidad de recidiva. Estos criterios si bien han permanecido en el tiempo no han sido validados. (Figuras 1-2 y 3).

El tratamiento definitivo es la resección quirúrgica completa, que puede efectuarse por toracotomía, videotoracoscopia (VTC) o cirugía videoasistida (VATS), dependiendo básicamente del tamaño y características del tumor.

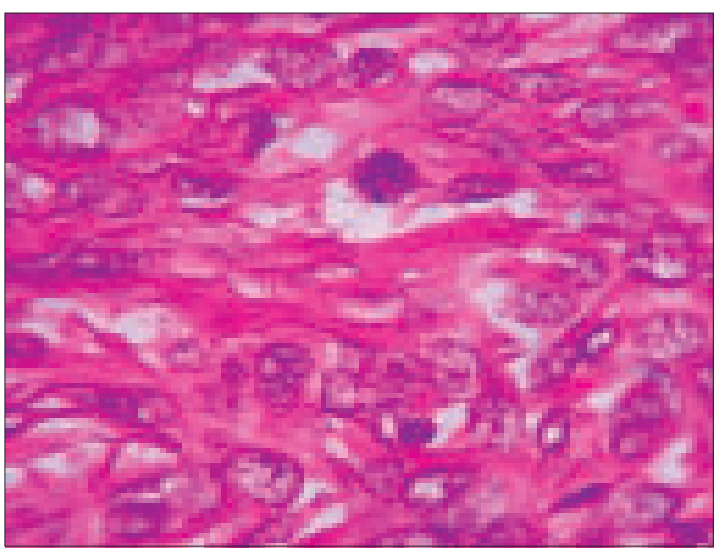

Figura 3. Lesión hipercelular con recuento mitótico alto. 
El diagnóstico de certeza y los criterios de malignidad deben establecerse en base al estudio histopatológico de la pieza operatoria.

El objetivo de este trabajo es mostrar la experiencia de nuestro Instituto en el manejo de estos tumores.

\section{Material y Métodos}

Revisamos retrospectivamente los archivos quirúrgicos del Instituto Nacional del Tórax (INT) entre marzo de 1991 y febrero de 2004, encontrando 41 casos de TFSP resecados.

A través de la revisión de fichas clínicas, radiología, protocolos operatorios y estudio histopatológico, confeccionamos un archivo que incluyó antecedentes personales y ambientales, sintomatología, motivo de consulta, hallazgos radiológicos y quirúrgicos, evolución postoperatoria y seguimiento. Para conceptos de seguimiento se consideró fecha de cierre del estudio el 31 de junio de 2004.

Desde el punto de vista histopatológico utilizamos como criterios de malignidad el hallazgo de cuatro o más mitosis por 10 campos de mayor aumento, la presencia de hemorragia y necrosis tumoral.

Todos los pacientes fueron estudiados con radiografía de tórax y tomografía computada (TC). Veinte pacientes $(48,7 \%)$ tenían fibrobroncoscopía y ocho $(19,5 \%)$ fueron sometidos a biopsia percutánea preoperatoria.

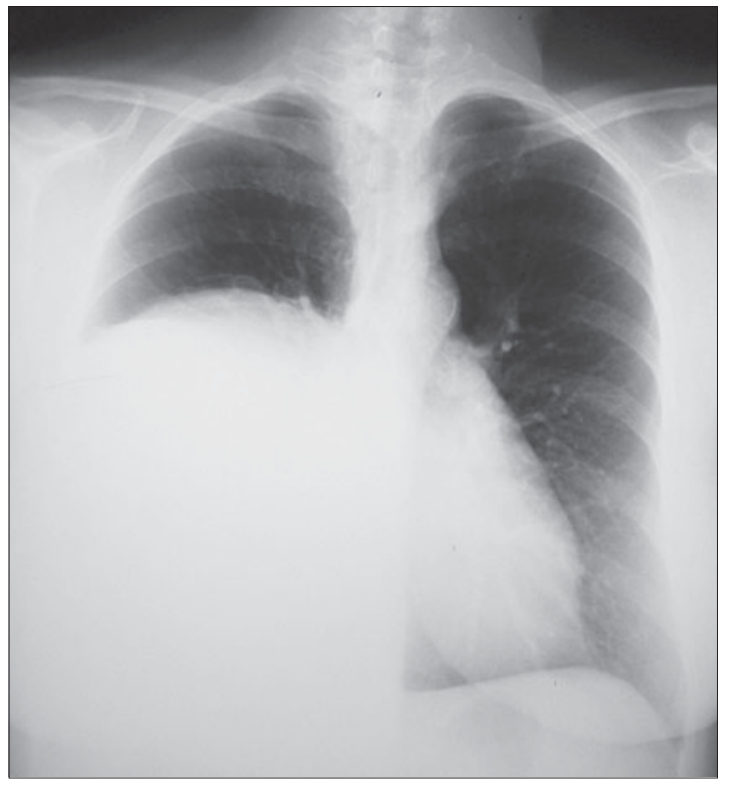

Figura 4. Gran masa que ocupa los dos tercios basales del hemitórax derecho.

\section{Resultados}

Veintisiete pacientes fueron mujeres $(65,9 \%)$ y catorce hombres $(34,1 \%)$. La edad media fue de 60,4 años (rango 31-79). Dieciocho pacientes $(43,9 \%)$ tenían antecedentes de tabaquismo y no se registró ninguno con exposición a asbesto.

En cuanto a la sintomatología, diez pacientes $(24,4 \%)$ fueron asintomáticos y el tumor fue un hallazgo radiográfico. Entre los sintomáticos, la tos y el dolor torácico fueron el principal motivo de consulta (Tabla 1). Durante el estudio en tres pacientes $(7,3 \%)$ se pudo detectar hipoglicemia. Todos ellos presentaban tumores mayores de $20 \mathrm{~cm}$ como diámetro mayor.

El hallazgo radiológico más frecuente fue una masa tumoral en 33 pacientes $(80,4 \%)$ (Figuras 4 y 5), seguido por imagen de nódulo pulmonar en $7(17,0 \%)$, y tumor mediastínico en $1(2,4 \%)$. Se asoció derrame pleural en 7 casos $(17,0 \%)$. El diagnóstico de TFSP se sospechó en 23 pacientes $(56,0 \%)$ mediante TC de tórax. Los otros diagnósticos preoperatorios se muestran en la Tabla 2.

Tabla 1. Motivo de consulta de 41 pacientes con tumor fibroso solitario de la pleura

\begin{tabular}{lcr}
\hline Motivo de consulta & $\begin{array}{c}\text { Número de } \\
\text { pacientes }\end{array}$ & \multicolumn{1}{c}{} \\
\hline Tos & 12 & 29,2 \\
Dolor torácico & 10 & 24,3 \\
Disnea & 8 & 19,5 \\
Hipoglicemia & 1 & 2,4 \\
Asintomáticos & 10 & 24,4 \\
Total & 41 & 100,0 \\
\hline
\end{tabular}

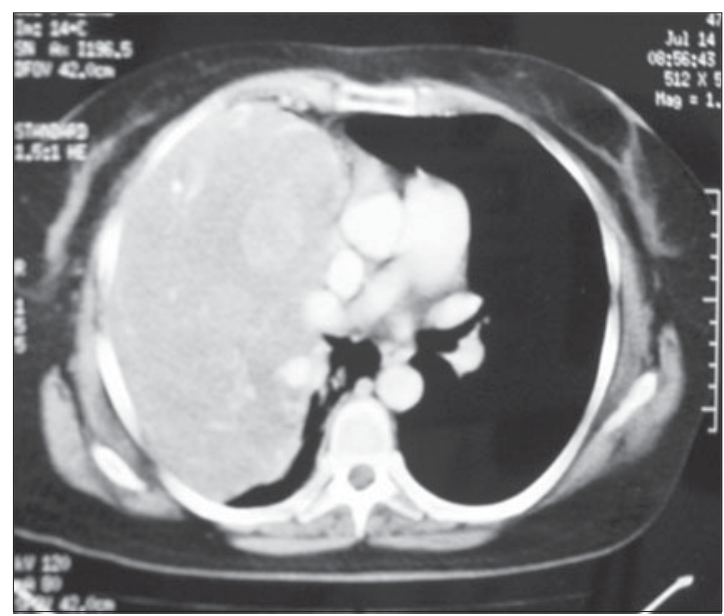

Figura 5. Tomografía de tórax en donde se muestra un tumor fibroso solitario de la pleura. 
Tabla 2. Diagnóstico preoperatorio de 41 pacientes con tumor fibroso solitario de la pleura

\begin{tabular}{lcr}
\hline $\begin{array}{l}\text { Diagnóstico } \\
\text { preoperatorio }\end{array}$ & $\begin{array}{c}\text { Número de } \\
\text { pacientes }\end{array}$ & $\%$ \\
\hline TFSP & 23 & 56,0 \\
Nódulo pulmonar & 7 & 17,0 \\
Cáncer pulmonar & 6 & 14,6 \\
Mesotelioma & 3 & 7,3 \\
Tumor mediastínico & 1 & 2,4 \\
Tumor de partes blandas & 1 & 2,4 \\
Total & 41 & 100,0 \\
\hline
\end{tabular}

Se realizó biopsia percutánea en 8 pacientes $(19,5 \%)$, confirmando el diagnóstico sólo en tres de ellos $(37,5 \%)$. La fibrobroncoscopía encontró signos de compresión extrínseca en 11 pacientes, sin otros signos patológicos.

Todos los pacientes fueron intervenidos quirúrgicamente, siendo abordados por toracotomía 36 de ellos (87,8\%), 3 mediante VTC (7,3\%) y 2 por VATS $(4,8 \%)$. La resección fue completa en todos los casos (Figura 6). La resección se asume completa dado que los tumores son encapsulados en su gran mayoría pero es imposible de asegurar en aquellos de gran tamaño con base de implantación extensa. En dos pacientes se debió complementar la resección con lobectomía por crecimiento intraparenquimatoso y destrucción lobar. En relación a la superficie de implantación, 31 tumores $(75,6 \%)$ se originaban de la pleura visceral y $10(24,4 \%)$ en la pleura parietal. Quince tenían pedículo $(36,5 \%)$, y los restantes presentaban base de implantación ancha.

La estadía postoperatoria media fue de 5,9 días (rango 2-24). El drenaje se mantuvo en promedio 3,8 días (rango 1-10). No hubo mortalidad operatoria y la morbilidad fue de un $12,1 \%$ (5 pacientes). En la Tabla 3 se muestra la morbilidad encontrada. Todos se manejaron médicamente excepto uno que requirió ser reintervenido por hemorragia.

El tamaño promedio de los tumores fue de $13,5 \mathrm{~cm}$ como diámetro mayor (rango $3-38 \mathrm{~cm}$ ),

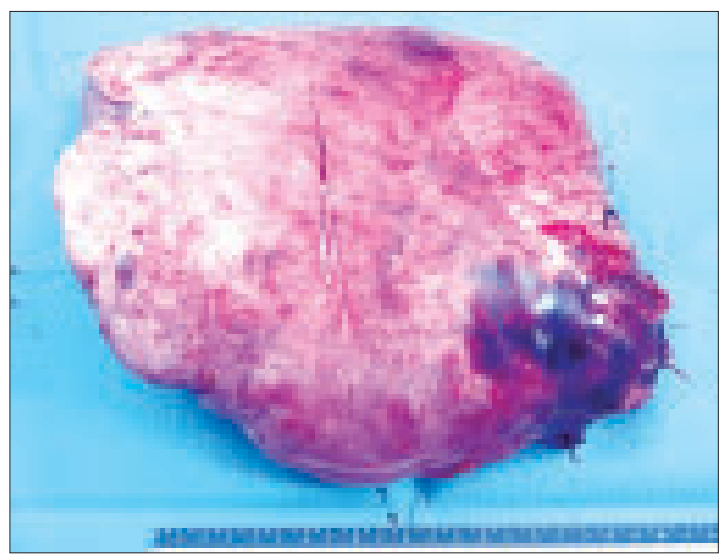

Figura 6. Apariencia macroscópica de un tumor fibroso solitario de la pleura resecado en forma completa.

Tabla 3. Morbilidad postoperatoria

\begin{tabular}{lcc}
\hline $\begin{array}{l}\text { Morbilidad } \\
\text { postoperatoria }\end{array}$ & $\begin{array}{c}\text { Número de } \\
\text { pacientes }\end{array}$ & \% \\
\hline Neumonia & 1 & 2,4 \\
Fístula broncopleural & 1 & 2,4 \\
Infección herida operatoria & 1 & 2,4 \\
Hemorragia postoperatoria & 1 & 2,4 \\
Neuritis intercostal & 1 & 2,4 \\
Total & 5 & 12,0 \\
\hline
\end{tabular}

y el peso promedio fue de $954 \mathrm{~g}$ (rango: 12$3.300 \mathrm{~g}$ ). El número de mitosis por 10 campos de mayor aumento se presentó en rango entre 0 y 6. En tres casos se encontraron 4 ó más mitosis. Necrosis del tumor se describe en 10 casos $(24,3 \%)$ y hemorragia en $9(22,0 \%)$.

El seguimiento promedio fue de 37,9 meses (rango 1-149 meses). Dentro del seguimiento se pudo detectar recidiva del tumor en tres pacientes $(7,3 \%)$, uno de los cuales recidivó en tres oportunidades. El tiempo entre la cirugía y el diagnóstico de la recidiva fue de 60,3 meses como promedio (rango 27-83 meses).

Se analizaron algunas características de los pacientes que recidivaron (Tabla 4).

Tabla 4. Características de pacientes con recidiva de tumor fibroso solitario de la pleura

\begin{tabular}{ccccccccccc}
\hline & Edad & Sexo & $\begin{array}{c}\text { Tamaño } \\
(\mathbf{c m})\end{array}$ & $\begin{array}{c}\text { Peso } \\
(\mathbf{g})\end{array}$ & Mitosis & Hemorragia & $\begin{array}{c}\text { Necrosis } \\
\text { tumoral }\end{array}$ & $\begin{array}{c}\text { Tiempo } \\
\text { recidiva }\end{array}$ & $\begin{array}{c}\text { Tratamiento Pedículo } \\
\text { recidivas }\end{array}$ \\
\hline 1 & 54 & F & 19 & 690 & 4 & $\mathrm{Sí}$ & $\mathrm{Si}$ & 27 meses & Resección & $\mathrm{Si}$ \\
2 & 64 & M & 17 & 760 & 6 & $\mathrm{Sí}$ & $\mathrm{Si}$ & 89 meses & Resección & $\mathrm{Si}$ \\
3 & 43 & F & 7 & 176 & 5 & No & No & 65 meses & Resección & No \\
\hline
\end{tabular}


Entre los pacientes que recidivaron, la histopatología demostró que todos presentaban 4 ó más mitosis por diez campo de mayor aumento, ninguno de los otros tumores presentó esta característica. Además, en dos de ellos se comprobó la presencia de hemorragia y necrosis tumoral en la muestra.

Todos los pacientes recidivados fueron resecados nuevamente. Uno de ellos presentó recidiva en tres ocasiones, siendo resecado en todas ellas, y falleció finalmente por infiltración masiva del mediastino, 81 meses posterior a la primera cirugía. Los otros dos pacientes se encuentran sanos y sin evidencias de nuevas recurrencias.

\section{Discusión}

Desde que Klemperer y Rabin en 1931 dividieron los tumores primarios de la pleura en difusos y localizados, los TFSP han adquirido diversos nombres hasta el actual. Es una neoplasia de crecimiento lento, y por ende poco sintomática, que puede alcanzar grandes dimensiones al momento del diagnóstico y suele ser un hallazgo en exámenes radiológicos de control. En nuestra serie casi un $25 \%$ de los pacientes fueron asintomáticos, siendo la tos, disnea y el dolor torácico las molestias más frecuentes, lo que concuerda con otras series ${ }^{6,11}$. No se ha demostrado relación alguna con el consumo de tabaco ni la exposición al asbesto $\mathrm{u}$ otros factores ambientales.

El diagnóstico preoperatorio suele sospecharse con las imágenes (radiografía y TC de tórax), siendo la presencia de una gran masa tumoral, homogénea y bien definida el hallazgo más frecuente $^{21}$.

La punción percutánea con aguja permite el diagnóstico histopatológico preoperatorio en algunos casos. En nuestra experiencia fue concordante sólo en tres casos de los ocho en que se realizó $(37,5 \%)$. Esta cifra concuerda con lo publicado por otros autores ${ }^{6,22}$, y por tanto creemos que su utilidad es relativa dado que la mayoría irá a cirugía resectiva de todos modos.

La hipoglicemia, que se encuentra en aproximadamente un 5 a $10 \%$ de los casos tendría relación con la producción de sustancias similares a la insulina por el tumor o la acción de inhibidores de la neoglucogénesis segregados por el tumor ${ }^{5-7,9-11,13-19}$. En nuestra serie encontramos hipoglicemia asociada al tumor en el 7,2\% de los pacientes.

La asociación con derrame pleural en general se presenta en alrededor del 10\% de los $\operatorname{casos}^{23}$, pero en nuestra serie fue algo mayor alcanzando un $17 \%$.

La mayoría de los tumores tienen su origen en la pleura visceral lo que se corroboró en nuestra serie. La presencia de pedículo tiene relación con el origen visceral del tumor y lo encontramos en el $36,5 \%$ de los casos. Los tumores que asientan en la pleura parietal son casi siempre de base ancha y no pediculados.

El tratamiento de elección es la cirugía resectiva completa y dependiendo del tamaño del tumor, la presencia de adherencias y el compromiso del parénquima adyacente, ésta puede realizarse por toracotomía, VATS o videotoracoscopía convencional.

En algunos casos, la extensión intralobar del tumor ("fibroma invertido") ${ }^{24}$, la destrucción del parénquima o la excesiva presencia de adherencias obligan a realizar resecciones segmentarias, lobectomías, e incluso neumonectomía para asegurar la excisión completa con buenos márgenes $^{6,11}$. Los tumores de base ancha que asientan en la pleura parietal a veces requieren de disección extrapleural, pero casi nunca es necesaria la resección concomitante de la pared torácica. En nuestra serie debimos complementar la resección con lobectomía en dos casos.

Pese a ser considerada una neoplasia benigna, en todas las series se describen recidivas $^{5,6,11,22,25}$, y pueden presentarse hasta 17 años posterior a la resección ${ }^{26}$. Tendrían mayor posibilidad de recurrir, aquellos tumores que presenten alguna de las características histológicas de malignidad descritas por England ${ }^{5}$. De estos criterios parecen ser más relevantes el número de mitosis y la necrosis pero es un punto que no está definido. Creemos que aquellos no pediculados podrían ser más susceptibles a la recidiva dada la gran superficie pleural sobre la que asientan lo que hace muy difícil asegurar microscópicamente los márgenes en forma completa. En nuestra serie tuvimos tres recurrencias $(7,3 \%)$, y todas ellas presentaron cuatro o más mitosis por 10 campos de mayor aumento. Además dos de los tres casos presentaron necrosis, hemorragia y eran de base ancha. Uno de nuestros casos recurrió en tres oportunidades, falleciendo finalmente 81 meses posterior a la primera cirugía. La recurrencia intratorácica puede ser fatal por compromiso mediastínico o de la vena cava ${ }^{20,27}$. Creemos que no hay índices claramente definidos para hablar de TFSP malignos y por tanto estos casos deben considerarse como recurrentes.

El rol de la terapia adyuvante no ha sido suficientemente estudiado dada la poca frecuencia 
de estos tumores, pero hay algunas comunicaciones aisladas ${ }^{23,28}$ que sugieren que la radioterapia y/o la quimioterapia podrían ser beneficiosos en algunos pacientes seleccionados.

En conclusión, el TFSP es una neoplasia infrecuente y de comportamiento generalmente benigno, pero puede recurrir e incluso ser fatal en un número reducido de casos. Creemos que aquellos tumores que presenten uno o más de los criterios de "malignidad" descritos por England et $\mathrm{al}^{5}$ deben ser seguidos en forma más acuciosa pues son los que tienen más posibilidades de recurrir. El único tratamiento efectivo es la resección quirúrgica completa, y en caso de recurrir deben ser nuevamente resecados.

\section{Bibliografía}

1.- KLEMPERER P, RABIN C B. Primary neoplasms of the pleura. A report of five cases. Arch Pathol 1931; 11: $385-412$.

2.- FLINT A, WEISS S W. CD-34 and keratin expression distinguishes solitary fibrous tumor (fibrous mesothelioma) of pleura from desmoplastic mesothelioma. Hum Pathol 1995; 26: 428-31.

3.- VAN DE RIJN M, LOMBARD C M, ROUSE R V. Expression of CD34 by solitary fibrous tumors of the pleura, mediastinum, and lung. Am J Surg Pathol 1994; 18: 814-20.

4.- WEYNAND B, NOEL H, GONCETTE L, NOIRHOMME P, COLLARD P. Solitary fibrous tumor of the pleura: a report of five cases diagnosed by transthoracic cutting needle biopsy. Chest 1997; 112: 1424-8.

5.- ENGLAND D M, HOCHHOLZER L, MCCARTHY M J. Localized benign and malignant fibrous tumors of the pleura. A clinicopathologic review of 223 cases. Am J Surg Pathol 1989; 13: 640-58.

6.- CARDILLO G, FACCIOLO F, CAVAZZANA A O, CAPECE G, GASPARRI R, MARTELLI M. Localized (solitary) fibrous tumors of the pleura: an analysis of 55 patients. Ann Thorac Surg 2000; 70: 1808-12.

7.- ROBINSON L A, REILLY R B. Localized pleural mesothelioma. The clinical spectrum. Chest 1994; 106: 1611-5.

8.- STRICKLAND-MARMOL L B, KHOOR A, ROBINSON L A, WILLIAMS C C Jr. Malignant localized fibrous tumor of the pleura. Cancer Control 2002; 9: 255-8.

9.- BLANCO M, MONTERO C, RIVAS J, OTERO I, YEBRA M T, VEREA H. Benign fibrous mesothelioma: report of 8 cases. Arch Bronconeumol 1998; 34: 361-3.

10.- ARIBAS O K, GORMUS N, KANAT F, AVUNDUK M C. Giant localized solitary fibrous tumors of the diaphragmatic pleura: report of two cases. Surg Today 2002; 32: 406-9.

11.- DE PERROT M, FISCHER S, BRUNDLER M A, SEKINE Y, KESHAVJEE S. Solitary fibrous tumors of the pleura. Ann Thorac Surg 2002; 74: 285-93.

12.- DORFMAN D M, TO K, DICKERSIN G R, ROSENBERG A E, PILCH B Z. Solitary fibrous tumor of the orbit. Am J Surg Pathol 1994; 18: 281-7.

13.- APPLE S K, NIEBERG R K, HIRSCHOWITZ S L. Fine needle aspiration biopsy of solitary fibrous tumor of the pleura. A report of two cases with a discussion of diagnostic pitfalls. Acta Cytol 1997; 41:
1528-33.

14.- SANDVLIET R H, HEYSTEEG M, PAUL M A. A large thoracic mass in a 57-year-old patient. Solitary fibrous tumor of the pleura. Chest 2000; 117: 897900.

15.- MOHAMED H, MANDAL A K. Natural history of multifocal solitary fibrous tumors of the pleura: a 25 year follow-up report. J Natl Med Assoc 2004; 96 : 659-62, 664.

16.- MOAT N E, TEALE J D, LEA R E, MATTHEWS A W. Spontaneous hypoglycaemia and pleural fibroma: role of insulin like growth factors. Thorax 1991; 46: 932-3.

17.- MASSON E A, MACFARLANE I A, GRAHAM D, FOY P. Spontaneous hypoglycaemia due to a pleural fibroma: role of insulin like growth factors. Thorax 1991; 46: 930-1.

18.- GARCÍA C E, BRAVO J L, ZAPATERO J. Masa gigante en el hemitórax derecho. Rev Patol Respir 2003; 6: 119-20.

19.- KHAN J H, RAHMAN S B, CLARY-MACY C, KERLAN R K, GEORGE T I, HALL T S, et al. Giant solitary fibrous tumor of the pleura. Ann Thorac Surg 1998; 65: 1461-4.

20.- BRISELLI M, MARK E J, DICKERSIN G R. Solitary fibrous tumors of the pleura: eight new cases and review of 360 cases in the literature. Cancer 1981; 47: 267889.

21.- VERSLUIS P J, LAMERS R J S. Localized pleural fibroma: radiological features. Eur J Radiology 1994; 18: $124-5$.

22.- DE PERROT M, KURT A M, ROBERT J H, BORISCH B, SPILIOPOULOS A. Clinical behavior of solitary fibrous tumors of the pleura. Ann Thorac Surg 1999; 67: 1456-9.

23.- SUTER M, GEBHARD S, BOUMGHARD M, PELOPONISIOS N, GENTON C Y. Localized fibrous tumours of the pleura: 15 new cases and review of the literature. Eur J Cardiothorac Surg 1998; 14: 453-9.

24.- YOUSEM S A, FLYNN S D. Intrapulmonary localized fibrous tumor: intraparenchymal so-called localized fibrous mesothelioma. Am J Clin Pathol 1988; 89: $365-9$.

25.- UTLEY J R, PARKER J C, HAHN R S, BRYANT L R, MOBIN-UDDIN K. Recurrent benign fibrous mesothelioma of the pleura. J Thorac Cardiovasc Surg 1973; 65: $830-4$

26.- OKIKE N, BERNATZ P E, WOOLNER L B. Localized mesothelioma of the pleura: benign and malignant variants. J Thorac Cardiovasc Surg 1978; 75: 363-72.

27.- DALTON W T, ZOLLIKER A S, MCCAUGHEY W T E, JACQUES J, KANNERSTAEIN M. Localized primary tumors of the pleura: an analysis of 40 cases. Cancer 1979; 44: 1465-75.

28.- VERONESI G, SPAGGIARI L, MAZZAROL G, DE PAS M, LEO F, SOLLI P, et al. Huge malignant localized fibrous tumor of the pleura. J Cardiovasc Surg 2000; 41: 781-4.

29.- LEZANA G, GONZÁLEZ S. Tumor fibroso pleural. Estudio inmunohistoquímico y ultraestructural de 14 casos. Rev Chil Enfer Respir 1994; 10: 87-93.

Correspondencia a:

Dr. Raimundo Santolaya Cohen

Instituto Nacional del Tórax

J. M. Infante 717 Providencia. Santiago, Chile.

Fax: 2360705

E-mail: rsantolaya@torax.cl 